\title{
Wilfrid Rall
}

\section{Gordon M. Shepherd ${ }^{1}$}

Published online: 23 May 2018

(C) Springer Science+Business Media, LLC, part of Springer Nature 2018

Wilfrid Rall (August 29, 1922 - April 1, 2018) was one of the founders of modern neuroscience. Trained as a biophysicist, he focused in the 1950s on the flow of electric current in neurons, and proved that the dendritic current dominated the integration of synaptic inputs and the generation of outputs. Ramon y Cajal (1852-1934) had provided the anatomical evidence for the elaborate structure of dendritic branching; Rall provided the rigorous functional framework for understanding the role of the dendritic structures and their electrical properties in neuronal function.

Rall was the son of Udo and Doris Rall and grew up in California. He received his B.S. in physics from Yale in 1942. He began graduate study in the new field of biophysics in Chicago, worked on the Manhattan Project during World War II, and completed his graduate studies in New Zealand with Archibald McIntyre during the time the department chair John Eccles was pioneering the first intracellular recordings from motor neurons. Returning to the U.S., in 1957 he joined the Mathematical Research Branch at the National Institutes of Health where he spent his career, retiring in 1994.

Stimulated by his studies in New Zealand, Rall founded two fields that have played central roles in the rise of modern neuroscience. His focus on how dendrites dominate the integrative properties of neurons made him the founder of the "Theoretical Foundation of Dendritic Function", the title of a volume containing his key articles on his retirement (https://mitpress.mit.edu/books/theoretical-foundationdendritic-function). The other field was computational neuroscience, whose founding document was his article written in 1962 (published in 1964) in the proceedings of

Gordon M. Shepherd

gordon.shepherd@yale.edu

1 Yale University, New Haven, CT 06520, USA a meeting held at Ojai, California, on new methods of theoretical brain science. In this article he laid out the compartmental method, in which segments of neuronal axons and dendrites were represented by equivalent circuits that could simulate passive, synaptic, or active properties in any arbitrary manner to test experimental results or predict new properties.

Pioneered first on spinal motor neuron dendrites, the Rall compartmental method was applied to brain neurons in the olfactory bulb, then extended by others in the 1970s to the cerebellar Purkinje cell, spinal cord interneurons, and hippocampal and neocortical pyramidal neurons. This method was then extended to study the spread out and into active dendrites and dendritic spines. With the transition in the 1980s to desktop computers and software packages such as NEURON and GENESIS, the Rall approach has become the standard for modeling neurons and neuronal circuits in realistic neuronal representations that bind theory with experiment. Around it has arisen other neuron modeling software (e.g. Matlab-based), digitization of neuronal morphology (Neurolucida), and analysis tools (e.g. exponent peeling).

Of particular interest for neuroinformatics, Rall models have been a primary stimulus to the construction of neuroinformatics databases for integrating multidisciplinary data into realistic representations of neurons and neuronal circuits. For example, NeuroMorpho.Org is a repository for the morphology of single neurons, which now includes over 86,000 examples. Neuroelectro.org archives the electrophysiological studies of over 70 neuron types for incorporation into Rall models. ModelDB archives over 1300 published computational models using Rall's compartmental modeling approach, providing the files for running the models or adapting them for new uses.

Rall initially had to overcome considerable resistance to his approach from anatomists who believed that neuronal morphology was too complicated to be modeled, and electrophysiologists who opposed the idea of widespread flow of current into and from dendritic branches. However, with 
the rise of computational neuroscience that he helped to found, Rall was repeatedly vindicated. He was a founding member of the Society for Neuroscience. In addition to being a member of the American Academy of Arts and Sciences, he was the first awardee of the Swartz Prize for
Theoretical and Computational Neuroscience, and received multiple nominations for the Nobel Prize. In addition to the significance of his work, Rall was a remarkably modest and genuine person, much beloved by all who worked with him or knew him. 